\title{
COMMENT
}

Check for updates Obesity and impaired metabolic
health in patients with COVID-19

Norbert Stefan ${ }^{1,2,3,4 凶}$, Andreas L. Birkenfeld ${ }^{1,2,3,5}$, Matthias B. Schulze $e^{3,6}$ and David S. Ludwig ${ }^{4,7,8}$

Preliminary data suggest that people with obesity are at increased risk of severe COVID-19. However, as data on metabolic parameters (such as BMI and levels of glucose and insulin) in patients with COVID-19 are scarce, increased reporting is needed to improve our understanding of COVID-19 and the care of affected patients.

IInstitute of Diabetes Research and Metabolic Diseases (IDM) of the Helmholtz Center Munich, Tübingen, Germany.

${ }^{2}$ Department of Internal Medicine IV, Division of Endocrinology, Diabetology and Nephrology, University Hospital of Tübingen, Tübingen, Germany.

${ }^{3}$ German Center for Diabetes Research (DZD), Neuherberg, Germany.

${ }^{4}$ Department of Pediatrics, Harvard Medical School, Boston, MA, USA

${ }^{5}$ Department of Diabetes, School of Life Course Science. King's College London, London, UK.

${ }^{6}$ Department of Molecular Epidemiology, German Institute of Human Nutrition Potsdam-Rehbruecke Nuthetal, Germany.

${ }^{7}$ Department of Nutrition, Harvard T.H. Chan School of Public Health, Boston, MA, USA.

${ }^{8} \mathrm{New}$ Balance Foundation Obesity Prevention Center, Boston Children's Hospital, Boston, MA, USA

凶e-mail:norbert.stefan@

med.uni-tuebingen.de

https://doi.org/10.1038 s41574-020-0364-6
In China, older age ( $\geq 65$ years) and the presence of comorbidities are associated with a more severe course of COVID-19 in patients infected with the novel coronavirus (SARS-CoV-2). Among the comorbid conditions, the highest fatality rate was found for cardiovascular disease (CVD) (10.5\%) and diabetes mellitus (7.3\%), followed by chronic respiratory diseases (6.3\%), hypertension $(6.0 \%)$ and cancer $(5.6 \%)^{1}$. A direct endocrine and metabolic link between hypertension and diabetes mellitus and coronavirus infection, which might involve angiotensin-converting enzyme 2, is being discussed $^{2}$.

\section{Early data}

Studies from China ${ }^{1}$ and the Lombardy region of Italy ${ }^{3}$ that have reported comorbidities in patients with COVID-19 did not provide data on body weight and height, which are used to estimate adipose tissue mass by calculating the BMI. A descriptive study of a small sample of 24 (63\% were men) critically ill patients diagnosed with COVID-19 in the Seattle region was among the first to report BMI data (3 patients with a BMI in the normal category, 7 with overweight, 13 with obesity and 1 with missing data). Although the numbers are too small for meaningful statistical analyses, $85 \%$ of the patients with obesity required mechanical ventilation and $62 \%$ of the patients with obesity died. These proportions are greater than those in the patients without obesity, in which $64 \%$ required mechanical ventilation and $36 \%$ died $^{4}$. As CVD and diabetes mellitus are strongly associated with elevated adipose tissue mass ${ }^{5}$, a high BMI might be an important risk factor for a severe course of disease, particularly of pneumonia, in these patients.

Concern about the effects of BMI is further substantiated by preliminary data from Shenzhen, China, and New York City, USA (the data have not been peer-reviewed). Among 383 patients from Shenzhen with COVID-19, overweight was associated with an $86 \%$ higher, and obesity with a $142 \%$ higher, risk of developing severe pneumonia compared with patients of normal weight in statistical models that controlled for potential confounders ${ }^{6}$. Among 4,103 patients with COVID-19 at an academic health system in New York City, BMI $>40 \mathrm{~kg} / \mathrm{m}^{2}$ was the second strongest independent predictor of hospitalization, after old age ${ }^{7}$. Furthermore, in a small study from a university hospital in Lille, France, reporting data from 124 patients with COVID-19, the need for invasive mechanical ventilation was associated with a BMI $\geq 35 \mathrm{~kg} / \mathrm{m}^{2}$, independently of other comorbidities ${ }^{8}$. The parameters mediating this high risk are thought to include impaired respiratory mechanics, increased airway resistance and impaired gas exchange, as well as other pathophysiological features of obesity, such as low respiratory muscle strength and lung volumes?.

\section{The obesity paradox}

Conversely, an obesity survival paradox has been observed in patients with pneumonia. That is, despite the increased risk of pneumonia and difficulties of intubation and mask ventilation, the risk of death in patients with obesity and pneumonia might be decreased ${ }^{10}$. Potentially counter-balancing effects of obesity might include the more aggressive treatment provided to these patients, their increased metabolic reserve or other unidentified factors ${ }^{10}$. Thus, as a result of a potentially critical role of body weight or adiposity in determining the incidence and severity of pneumonia (and possibly other complications), it is important to collect anthropometric information for patients with COVID-19.

Furthermore, the impaired metabolic health (characterized by hypertension, dyslipidaemia and hyperglycaemia) associated with obesity might also be present in those with normal weight or overweight ${ }^{5}$. Prediabetes, which is present in $38 \%$ of the adult population in the USA, was identified as an important risk factor for CVD and renal disease ${ }^{5}$. To what extent these cardiometabolic risk factors predispose individuals to severe disease independently of BMI remains to be determined. 


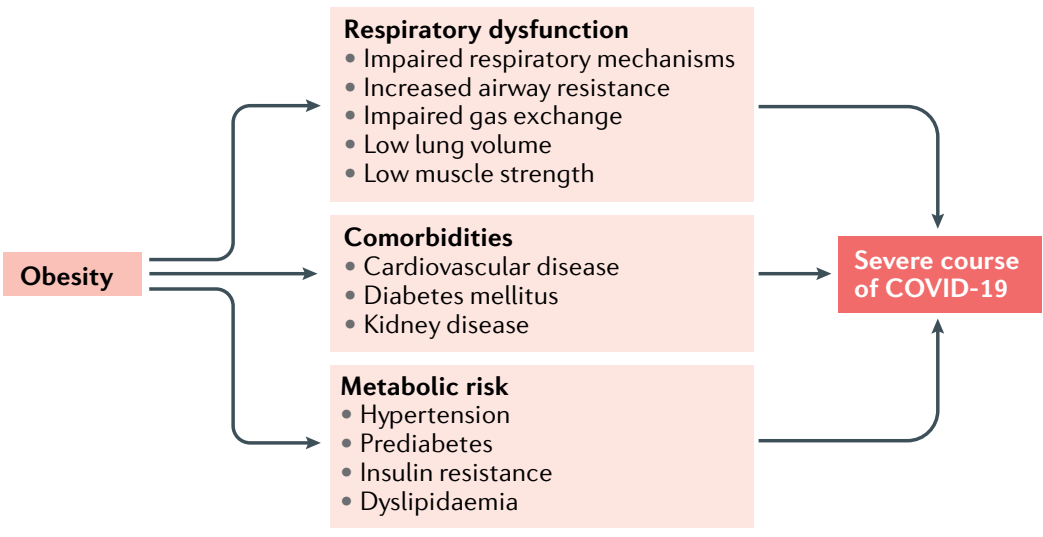

Fig. 1 | Obesity-related comorbidities and mechanisms of a severe course

of COVID-19. Patients with obesity often have respiratory dysfunction, which is characterized by alterations in respiratory mechanisms, increased airway resistance, impaired gas exchange and low lung volume and muscle strength. These individuals are predisposed to hypoventilation-associated pneumonia, pulmonary hypertension and cardiac stress. Obesity is also associated with an increased risk of diabetes mellitus, cardiovascular disease and kidney disease, comorbidities that are considered to result in increased vulnerability to pneumonia-associated organ failures. However, even in the absence of comorbidities of obesity, the presence of hypertension, dyslipidaemia, prediabetes and insulin resistance might predispose individuals to cardiovascular events and increased susceptibility to infection via atherosclerosis, cardiac dysfunction and impaired immune response.
Such measurements might be useful both in a primary care setting and in a hospital setting to assess the risk of a complicated course of disease in patients with a positive SARS-CoV-2 test (FIG. 1).

In conclusion, while it is widely recognized that the presence of comorbidities such as hypertension, diabetes mellitus and CVD is associated with a more severe course of COVID-19, obesity has hardly been investigated so far. Obesity is a main risk factor for these comorbidities and more generally for impaired metabolic health (such as dyslipidaemia and insulin resistance) and is also linked to an increased risk of pneumonia. Measurement of anthropometric characteristics and metabolic parameters is crucial to better estimate the risk of complications in patients with COVID-19.

1. Wu, Z. \& McGoogan, J. M. Characteristics of and important lessons from the coronavirus disease 2019 (COVID-19) outbreak in china: summary of a report of 72,314 cases from the Chinese Center for Disease Control and Prevention. JAMA 323 1239-1242 (2020)

2. Bornstein, S. R., Dalan, R., Hopkins, D., Mingrone, G. $\&$ Boehm, B. O. Endocrine and metabolic link to coronavirus infection. Nat. Rev. Endocrinol. https://doi.org/10.1038/ s41574-020-0353-9 (2020).

3. Grasselli, G. et al. Baseline characteristics and outcomes of 1591 patients infected with SARS-CoV-2 admitted to ICUs of the Lombardy Region, Italy. JAMA https://doi.org/10.1001/ jama.2020.5394 (2020).

4. Bhatraju, P. K. et al. Covid-19 in critically ill patients in the Seattle region-Case Series. N. Engl. J. Med. https://doi.org/10.1056/ NEJMoa2004500 (2020).

5. Stefan, N., Schick, F. \& Häring, H. U. Causes, characteristics, and consequences of metabolically unhealthy normal weight in humans. Cell Metab. 26, 292-300 (2017).

6. Qingxian, C. et al. Obesity and COVID-19 severity in a designated hospital in Shenzhen, China. Preprint at SSRN https://doi.org/ 10.2139/ssrn.3556658 (2020).

7. Petrilli, C. M. et al. Factors associated with hospitalization and critical illness among 4,103 patients with COVID-19 disease in New York City. Preprint at medRxiv https://doi.org/10.1101/ 2020.04.08.20057794 (2020).

8. Simonnet, A. et al. High prevalence of obesity in severe acute respiratory syndrome coronavirus-2 (SARS-CoV-2) requiring invasive mechanical ventilation. Obesity https://doi.org/10.1002/oby.22831 (2020).

9. Murugan, A. T. \& Sharma, G. Obesity and respiratory diseases. Chron. Respir. Dis. 5, 233-242 (2008).

10. Nie, W. et al. Obesity survival paradox in pneumonia: a meta-analysis. BMC Med. 12, 61 (2014).

\section{Competing interests}

D.S.L. has received royalties for books on nutrition and obesity. The other authors declare no competing interests. 